\title{
Apurinic/apyrimidinic endonuclease 1 (APE1) contributes to resveratrol-induced neuroprotection against oxygen-glucose deprivation and re-oxygenation injury in HT22 cells: Involvement in reducing oxidative DNA damage
}

\author{
JIAO-YING JIA, ZHI-GANG TAN, MIN LIU and YU-GANG JIANG
}

Department of Neurosurgery, The Second Xiangya Hospital of Central South University, Changsha, Hunan 410011, P.R. China

Received March 8, 2017; Accepted September 19, 2017

DOI: $10.3892 / \mathrm{mmr} .2017 .7799$

\begin{abstract}
Resveratrol, a naturally occurring polyphenolic compound, exhibits a neuroprotective role in models of central nervous system diseases, including cerebral ischemia/reperfusion injury. Apurinic/apyrimidinic endonuclease 1 (APE1) is a multifunctional enzyme that contributes to base excision repair of oxidative DNA damage and redox activation of transcription factors, associated with neuronal survival against hypoxic-ischemic injury. It was hypothesized that resveratrol protects HT22 cells against oxygen-glucose deprivation and re-oxygenation (OGD/R)-induced injuries through upregulation of APE1. It was demonstrated that resveratrol pretreatment significantly increased the viability of HT22 cells and decreased the release of lactate dehydrogenase (LDH), accompanied by the upregulation of APE1 mRNA, and protein levels, as well as the activity of APE1 under $\mathrm{OGD} / \mathrm{R}$ conditions. In addition, resveratrol reversed OGD/R-induced oxidative DNA damage as evidenced by the decreases in the levels of 8-hydroxy-2'-deoxyguanosine and APE sites. However, APE1 knockdown using short hairpin RNA sequence targeting APE1 abolished resveratrol-elicited beneficent effects against OGD/R-induced cytotoxicity and oxidative stress. This was indicated by decreased cell viability, superoxide dismutase activity and glutathione levels, and increased LDH release and reactive oxygen species levels. The results of the present study indicate that APE1 contributes to the protective effects of resveratrol against neonatal hypoxic-ischemic brain injuries, and suggest that DNA repair enzymes, including APE1, may be a unique strategy for neuroprotection against this disease.
\end{abstract}

Correspondence to: Dr Yu-Gang Jiang, Department of Neurosurgery, The Second Xiangya Hospital of Central South University, 139 Renmin Middle Road, Changsha, Hunan 410011, P.R. China E-mail: yugjiang@foxmail.com

Key words: Apurinic/apyrimidinic endonuclease 1, resveratrol, neuroprotection, cerebral ischemia injury, oxidative DNA damage

\section{Introduction}

Cerebral ischemia injury is a common and serious neurological disease, leading to causes of death long-term disability worldwide (1). Despite hundreds of preclinical trials demonstrating efficacy of neuron-targeted therapies in vivo and vitro models of stroke, the only clinical treatment remains early restoration of blood flow with thrombolysis (2). The failure to translate neuron-targeted approaches to beneficent clinical therapy indicates that alternative cellular targets in brain may more effectively coordinate the complex intracellular and extracellular signaling cascades which contribute to neuronal injury. Emerging evidences reveal that one of the most widely accepted pathophysiological mechanisms of cerebral ischemia involve oxidative DNA damage $(3,4)$.

Oxidative DNA damage is an early event following cerebral ischemia-reperfusion injury, resulting from direct or indirect attacks by reactive oxygen species (ROS) during reperfusion (4-6). Oxidative DNA damage consists of DNA-protein crosslinks, 8-hydroxy-2 $\phi$-deoxyguanosine (8-OHdG) formation and apurinic/apyrimidinic (AP) sites $(4,5)$. Apurinic/apyrimidinic endonuclease 1 (APE1) is a multifunctional enzyme that participates in base-excision repair of oxidative DNA damage and in the redox activation of transcription factors $(7,8)$. Neurons with decreased APE1 expression and endonuclease activity were found to be extremely vulnerable to cell death induced by in vitro ischemia, indicating that oxidative base lesions and AP sites can trigger ischemic cell death (9). Furthermore, a strong correlation exists between loss of APE1 expression in ischemic neurons and neuronal cell death after ischemia $(10,11)$. Energy failure after ischemia has been speculated to deplete APE1 expression, thereby triggering neuronal death (12). In another study, APE1 is required for pituitary adenylate cyclase-activating polypeptide (PACAP)-induced neuroprotection against global cerebral ischemia (13). However, the role of endogenous APE1 in cellular protection from ischemic injury has not been unequivocally established.

Resveratrol (3,5,4'-trihydroxy-trans-stilbene) is a naturally occurring phytoalexin that is found abundantly in the skin of red grapes and is a component of red wine (14). Several studies have demonstrated that resveratrol elicits a variety of 
biological and pharmacological functions, including cardioprotective, anti-oxidant, anti-apoptotic, and anti-inflammatory activities (15-18). In addition, resveratrol is also regarded as a natural antioxidant, has been suggested to reduce DNA damage and oxidative organ injury (19). Importantly, increasing studies have identified a neuroprotective role of resveratrol in animal models of cerebral ischemia/reperfusion injury (20-22). However, the potential neuroprotective effects of resveratrol against hypoxic-ischemic brain injury and the underlying mechanisms remain clear unknown.

Therefore, the present study attempts to investigate the role of APE1 in the protective effect of resveratrol against oxygen-glucose deprivation and re-oxygenation (OGD/R)-induced HT22 cells injury which is an effective in vitro model of cerebral ischemia. We found that resveratrol prevents OGD/R-induced cytotoxicity and oxidative stress accompanied by increasing APE1 activity and protein and mRNA levels, resulting in neuroprotective effect under cerebral ischemic environment, while these effects are blocked by APE1 shRNA. The present study reveals a central role of DNA repair induced by APE1 in resveratrol-induced neuroprotection after cerebral ischemia.

\section{Materials and methods}

Reagents. Resveratrol (RSV) was purchased from Sigma-Aldrich (St. Louis, MO, USA). Dulbecco's Modified Eagle's Medium (DMEM) and fetal bovine serum (FBS) were obtained from GIBCO Life Technologies (Paisley, Scotland). 3-(4,5-dimethylthiazol-2-yl)-2,5-diphenyltetrazolium

bromide (MTT) assay kit (C0009) and lactate dehydrogenase (LDH) cytotoxicity assay kit (C0016) were from Beyotime Institute of Biotechnology (Jiangsu, China). The primary antibodies against APE1, tubulin and HRP conjugated goat anti-rabbit IgG were obtained from Cell Signaling Technology (Beverly, MA, USA). ROS detection reagent, 5-(and-6)-carboxy-2', 7'-dichlorodihydrofluorescein diacetate (carboxy- $\mathrm{H}_{2}$ DCFDA, C400), was purchased from Invitrogen (Carlsbad, CA, USA).

Cell culture. HT22 cells were obtained from the National Cell Bank of Iran (Pasteur Institute of Iran, Tehran, Iran). The cells were cultured in DMEM containing 10\% FBS, $100 \mathrm{U} / \mathrm{ml}$ of penicillin and $100 \mu \mathrm{g} / \mathrm{ml}$ of streptomycin under normal culture condition $\left(5 \% \mathrm{CO}_{2}\right.$ and $95 \%$ humidified air at $\left.37^{\circ} \mathrm{C}\right)$.

Oxygen-glucose Deprivation and Re-oxygenation (OGD/R) model. After grown to $60-70 \%$ confluency in a standard cell culture incubator (humidified $5 \% \mathrm{CO}_{2}$ athmosphere and $37^{\circ} \mathrm{C}$ ), the HT22 cells were exposed to OGD/R process. Briefly, cultured medium was instead of glucose-free Neurobasal A medium (Life Tech, USA) and the cells were put in a hypoxic chamber at $37^{\circ} \mathrm{C}$ with a mix gas containing $1 \% \mathrm{O}_{2}, 5 \% \mathrm{CO}_{2}$ and $94 \% \mathrm{~N}_{2}$, which was monitored with O2analyzer (GODEE, China). After incubation for $2 \mathrm{~h}$, cells were returned to normal cultured conditions for re-oxygenation ( $48 \mathrm{~h}$ ) according to the instructions of the manufacturers.

Constructs and transfection. The lentiviral vectors expressing APE1-targeting sequence (APE1t) or the scrambled sequence
(APE1s) were constructed using pDC315-eGFP adenovirus vector. shRNA sequence for huamn APE1 were inserted into pDC315-eGFP adenovirus vector. The recombinant adenoviral vector (pDC315-eGFP-APE1 shRNA and pDC315-eGFP-APE1 scramble) was packaged and amplified in HEK 293A cells. The following oligonucleotides were used for the APE1 shRNA: sense, 5'-GATCCCCCCTGCCACACT CAAGATCTGCTTCAAGAGAGCAGATCTTGAGTGTGG CAGGTTTTTGGAAA-3'; and antisense,5'-AGCTTTTCC AAAAACCTGCCACACTCAAGATCTGCTCTCTTGAAG CAGATCTTGAGTGTGGCAGGGGG-3'; and scrambled oligonucleotide sequences: Sense, 5'-GATCCCCAGTCTAA CTCGCCACCCCGTATTCAAGAGATACGGGGTGGC GAGTTAGACTTTTTTGGAAA-3'; antisense, 5'-AGC TTTTCCAAAAAAGTCTAACTCGCCACCCCGTATCT CTTGAATACGGGGTGGCGAGTTAGACTGGG-3'. The transfections were performed using Lipofectamine ${ }^{\mathrm{TM}} 3000$ Transfection Reagent (L3000001, Invitrogen) following the manufacturer's protocol for transient transfection of HT22 cells. The transfection efficiency was detected using Real-time quantitative PCR (RT-PCR) and western blot analysis.

Cell viability assay. HT22 cell survival was measured using a MTT assay. At the end of the incubation period, the culture medium was removed and HT22 cells were incubated with MTT solution $(0.5 \mathrm{mg} / \mathrm{ml})$ in a dark place for $4 \mathrm{~h}$ at $37^{\circ} \mathrm{C}$. Then, cells were treated with dimethyl sulfoxide (DMSO) in order to dissolve the formazan crystals. Absorbance at $595 \mathrm{~nm}$ was assessed using a microplate reader (PowerWave XS; BioTek Instruments, Wincoski, VT, USA). Viability was assessed by comparison of the absorbance of different treatment of the samples with control group according to the following formula and each experiment was carried out for triplicate: Cell viability $(\%)=\left(\mathrm{OD}_{\text {experimental group }}-\mathrm{OD}_{\text {black }} / \mathrm{OD}_{\text {control }}\right.$ group $\left.-\mathrm{OD}_{\text {black }}\right)$ x $100 \%$.

LDH assay. The cytotoxicity of HT22 cells following the exposures was assessed by measuring the amount of released lactate dehydrogenase (LDH) enzyme from cells using lactate dehydrogenase (LDH) cytotoxicity assay kit according to the manufacturer's instructions. In brief, HT22 cells were seeded into 6 -well plate at a density of $1 \times 10^{6}$ cells/well. At the end of the treatment, LDH from the culture medium was measured at $340 \mathrm{~nm}$. The cells were lysed in $0.5 \mathrm{ml}$ of lysis buffer provided within the assay. The amount of intracellular LDH released into the extracellular medium is expressed as a fold of total LDH activity ( $\mathrm{LDH}$ in the medium $+\mathrm{LDH}$ in the cell) according to the following equation: $\mathrm{LDH}$ released $=\mathrm{LDH}$ activity in the medium/total LDH activity.

Measurement of APE1 levels using ELISA kit. The culture supernatant of HT22 cells treated with indicated regents were collected. Prepare and mix all reagents thoroughly before use. Sample or standard $(50 \mu \mathrm{l})$ were added to the wells of the conjugate coated plate, respectively. After incubated for $10 \mathrm{~min}$ at room temperature, the diluted anti-APE1 antibody $(50 \mu \mathrm{l})$ was added to each well and co-incubated at room temperature for $1 \mathrm{~h}$. After washed three times with 1x Wash Buffer $(250 \mu \mathrm{l})$ per well with thorough aspiration between 
each wash, the diluted secondary antibody-enzyme conjugate (100 $\mu 1)$ was added to all wells and incubated at room temperature for $1 \mathrm{~h}$. Following washed three times, warm substrate solution $(100 \mu \mathrm{l})$ was added to each well and incubated at room temperature for $30 \mathrm{~min}$. Finally, stop solution $(100 \mu \mathrm{l})$ was added into each well plate to stop the enzyme reaction. Results should be read immediately at $450 \mathrm{~nm}$ wave length by Mithras LB 940 Multimode Microplate Reader (Berthold Technologies GmbH \& Co., Bad Wildbad, Germany).

8-hydroxydeoxyguanosine (8-OHdG) level measurement. 8-OHdG, a marker of oxidative stress to DNA, was also measured by an enzyme linked immunosorbent assay (ELISA; YLA0016HU; Hangzhou Eastbiopharm Co., Ltd., Hangzhou, China), according to the manufacturer's instructions. The absorbance was measured at $450 \mathrm{~nm}$. The results were represented as the number of $8-\mathrm{OHdG}$ per $10^{6}$ nucleotides.

AP Sites of DNA measurement. Nuclear DNA of HT22 cells was freshly isolated after treatment for indicated time. AP sites were tested by the colorimetric assay kit (CAS:154-21-2; Integrated Device Technology, San Jose, CA, USA). Biotin labeled aldehyde reactive probe (ARP) in the ring-opened AP site was detected for AP sites. The purified DNA of HT22 cells was dissolved in TE at $100 \mu \mathrm{g} / \mathrm{ml}$, and then DNA solution $(10 \mu \mathrm{l})$ was incubated with ARP solution $(5 \mathrm{mM}, 10 \mu \mathrm{l})$ at $37^{\circ} \mathrm{C}$ for $1 \mathrm{~h}$. The ARP-labeled DNA was then precipitated with ethanol, and the DNA pellet was resuspended in TE. ARP in the labeled DNA was measured using an ELISA-like assay in a microtiter plate according to the manufacturer's instructions. OD values of each well were measured by Mithras LB 940 Multimode Microplate Reader (Berthold Technologies $\mathrm{GmbH} \&$ Co.) at $650 \mathrm{~nm}$. All ARP assays were performed in triplicate. The results were represented as the number of AP sites per $10^{6}$ nucleotides, were normalized with calibration curve based on ARP-DNA standard solutions.

Intracellular reactive oxygen species (ROS) measurement. ROS generation was determined using the oxidative conversion of cell-permeable DCFH-DA to fluorescent DCF. HT22 cells were cultured in 6-well plates at a density of $1 \times 10^{6}$ cells/well. After indicated treatments, cells were harvested, resuspended in $1 \mathrm{ml}$ PBS with $20 \mu \mathrm{M}$ of carboxy-H2DCFDA, and then incubated for $1 \mathrm{~h}$ at $37^{\circ} \mathrm{C}$. After washing, DCF fluorescence was measured using a Coulter CyFlow Cytometer (Partec). The results were expressed as the mean DCFH-DA fluorescence intensity over that of the control.

Cellular SOD and GSH activity analysis. Oxidative stress damage is prevented by the rapid scavenging of $\mathrm{O}^{-}$by the mitochondrial enzyme manganese superoxide dismutase (SOD). Glutathione (GSH) is an intracellular antioxidant and plays an important role in the detoxification of various electrophilic compounds. The activity of SOD and the level of GSH were detected using SOD activity kit (KT-219) and GSH measurement kit (K251-100) supplied by Assay Designs (Ann Arbor, MI, USA), respectively. Briefly, HT22 Cells were seeded in 6-well plates $\left(1 \times 10^{6}\right.$ cells/well $)$ and subjected to the treatment. Then, the cells were harvested and protein was extracted.
SOD activity was measured by adding the master mix and xanthine supplied in the kit, followed by incubation and measurement by ELISA reader at $450 \mathrm{~nm}$ for $10 \mathrm{~min}$ at 1 -min intervals. GSH was determined by adding the reaction mix and GSH reductase supplied in the kit, followed by incubation and measurement by ELISA reader at $405 \mathrm{~nm}$ for $20 \mathrm{~min}$ at 1-min intervals. Protein concentration was quantified by using a Bio-Rad protein assay kit. Then, SOD activity in the cell extracts was calculated vs. a SOD standard curve and normalized to the protein concentration. The total amount of GSH was determined by means of a calibration curve and normalized to the protein concentration.

Real Time RT-PCR. Total RNA was extracted and purified from treated HT22 cells using the RNA isolator Total RNA Extraction Reagent (TaKaRa, Kusatsu, Japan) in accordance with manufacturer's instructions. Total RNA was subjected to reverse transcription using iScript ${ }^{\mathrm{TM}}$ cDNA Synthesis kit and Real-time quantitative PCR (RT-PCR) was performed according to the AceQ ${ }^{\circledR}$ qPCR SYBR ${ }^{\circledR}$ Green Master Mix kit (TaKaRa) on ABI 7500 system (ABI, New York, NY, USA). Glyceraldehyde-3-phosphate dehydrogenase (GAPDH) was used to normalize levels of specific mRNA between samples. Primer pairs were designed as follows: APE1: 5'-CTGCCT GGACTCTCTCATCAATAC-3' and 5'-GAATGCCGTATC CGCTACTCC-3'; GAPDH: 5'-GCACCGTCAAGGCTGAGA AC-3', GAPDH R: 5'-TGGTGAAG ACGCCAGTGGA-3'. Relative quantification of the indicated mRNA normalized against GAPDH mRNA was calculated by using the $2^{-\Delta \Delta C T}$ methods.

Western blot analysis. The cells were homogenized with protein extraction solution (lysis in RIPA). The homogenate was centrifuged at $12,000 \mathrm{rpm}$ at $4^{\circ} \mathrm{C}$ for $30 \mathrm{~min}$ and total protein were quantified by using a Bio-Rad protein assay kit. Equal amounts of proteins were separated by $12 \%$ sodium dodecyl sulfate-polyacrylamide gel electrophoresis (SDS-PAGE) and then transferred to polyvinylidene fluoride membranes (PVDF, Millipore, Billerica, MA, USA). After blocked with 5\% skim milk in PBST for $2 \mathrm{~h}$ at room temperature, followed by overnight exposure to primary antibody against APE1 (cat. no. 4128, 1:2,000; Cell Signaling Technology) or -tubulin (cat. no. 5335, 1:2,000; Cell Signaling Technology). Membranes were then incubated with appropriate horseradish peroxidase (HRP)-conjugated antibody (cat. no. 7074, 1:5,000; Cell Signaling Technology) for $2 \mathrm{~h}$ at room temperature. Tubulin was performed as an internal loading control. Bands were visualized using an enhanced chemiluminescence system (Pierce Biotech, Rockford, IL, USA) and imaged using ImageJ software (NIH). Results are representative of at least three experiments and defined as the percentage of the control group after being normalized against tubulin.

Statistical analysis. Statistical analyses were conducted with GraphPad Prism 5 (Graphpad Software, San Diego, CA, USA) and expressed as the mean \pm standard deviation (SD). Differences between the groups were analyzed with the one-way ANOVA test followed by Dunnett's post hoc tests. $\mathrm{P}<0.05$ was considered significant. 
A

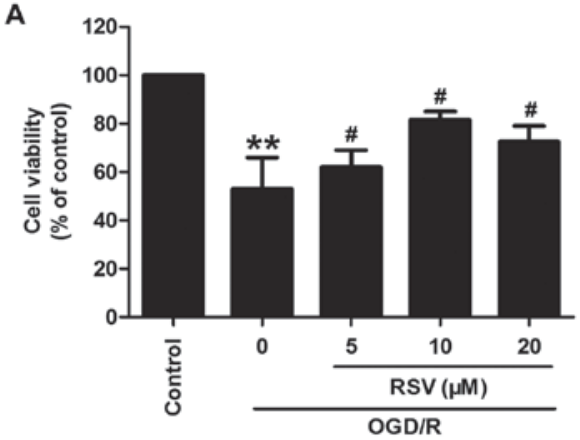

C

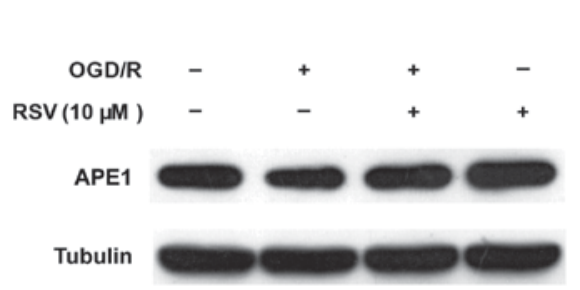

E

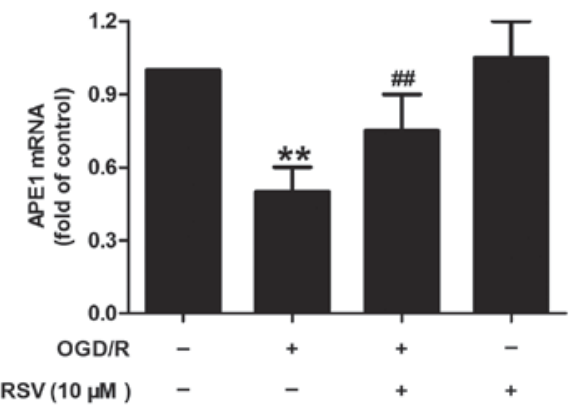

B
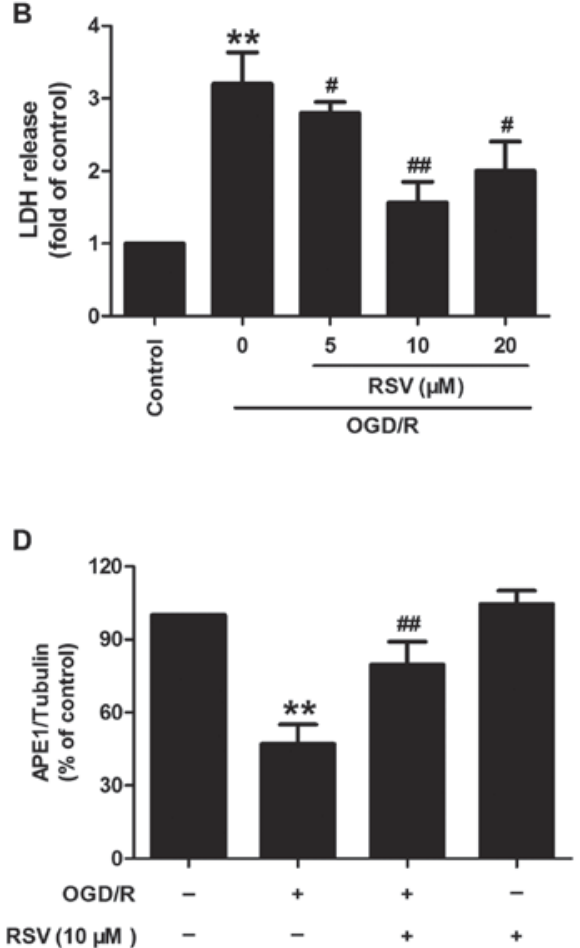

$\mathbf{F}$

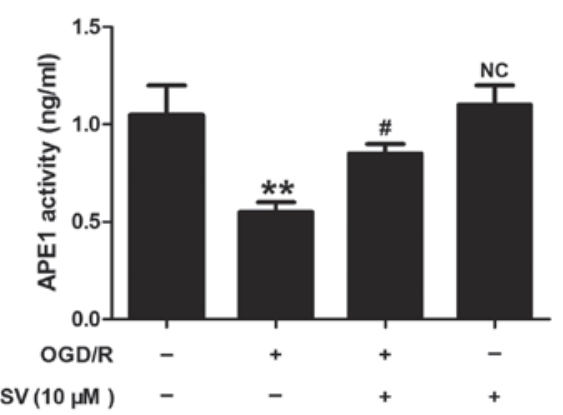

Figure 1. Resveratrol increases APE1 level and activity and decreases cytotoxicity in HT22 cells after oxygen-glucose deprivation and re-oxygenation (OGD/R) treatment. HT22 cells were pretreated with resveratrol $(5,10$ or $20 \mu \mathrm{M})$ for $30 \mathrm{~min}$ and then subjected to OGD/R process as shown in 'Materials and methods'. (A) The viability of cells was measured by MTT assay. (B) The LDH release was measured by lactate dehydrogenase (LDH) cytotoxicity assay kit. (C) The expression of APE1 was determined using western blot assay and (D) Quantitative analysis of western blot results assay. Densitometry analyses are presented as the relative ratio of protein/tubulin, and data are presented as percentages of control group. (E) The level of APE1 mRNA was measured by Real-time quantitative PCR (RT-PCR). Results were normalized to control levels. (F) The activity of APE1 in culture supernatant was detected using ELISA kit. Data are expressed as fold changes normalized to the control group. Bars represent the means $\pm \mathrm{SD}$ from three independent experiments. ${ }^{* *} \mathrm{P}<0.01 \mathrm{vs}$. control group; ${ }^{\#} \mathrm{P}<0.05 ;{ }^{\#} \mathrm{P}<0.01$ vs. OGD/R treatment alone group. RVS, resveratrol; OGD/R, oxygen-glucose deprivation and re-oxygenation, NC: No statistical significance.

\section{Results}

Resveratrol decreases neurotoxicity and increases APE1 activity and APE1 levels in oxygen-glucose deprivation and re-oxygenation $(O G D / R)$-treated HT22 cells. Firstly, we investigated whether resveratrol (RSV) had protection and influence on APE1 in OGD/R-treated HT22 cells. As shown in Fig. 1, OGD/R condition induced the decrease in cell viability (Fig. 1A) and the increase in LDH release (Fig. 1B) in HT22 cells. However, the effects were reversed by different doses of $\operatorname{RSV}(5,10$, and $20 \mu \mathrm{M})$ pretreatment. The cell viability significantly increased in response to the concentration of RSV $(10 \mu \mathrm{M})$ which may reach saturation values compared to RSV $(20 \mu \mathrm{M})$, so $10 \mu \mathrm{M}$ of RSV was selected as the optimal concentration for subsequent experiments to demonstrate the effect of RVS on APE1 level and activity in HT22. Next, western blot analysis result (Fig. 1C) reveled that RVS (10 $\mu \mathrm{M})$ pretreatment mitigated OGD/R-induced the down-regulation of APE1 protein level in HT22 cells (Fig. 1D). In addition, RVS pretreatment also abolished the decreases in the level of APE1 mRNA (Fig. 1E) and the activity of APE1 (Fig. 1F) induced by OGD/R treatment in HT22 cells. RVS treatment alone had no effect on the activity and level of APE1. Take together, these results suggested that APE1 may contribute to the protective effects of RVS against OGD/R-induced nerve damage.

Resveratrol alleviates $O G D / R$-induced oxidative DNA damage and repair activity in HT22 cells. APE1 is a multifunctional enzyme that plays a part in base-excision 
repair of oxidative DNA injury and in the redox activation of transcription factors (7). To further determine the role of APE1 in RVS-elicited beneficent effect on cerebral ischemia, we detected the levels of 8 -hydroxy-2 $\phi$-deoxyguanosine (8-OHdG) and apurinic/apyrimidinic (AP) sites which were considered as oxidative DNA damage markers. As illustrated in Fig. 2, OGD/R treatment significantly increased the levels of 8-OHdG (Fig. 2A) and AP sites (Fig. 2B) in HT22 cells, while these effects were obviously blocked by RVS $(10 \mu \mathrm{M})$ pretreatment. RVS itself did not induce the changes of 8-OHdG and AP sites levels. These results indicated that RSV alleviates OGD/R-induced oxidative DNA damage and repair activity, which may mean up-regulation of APE1 activity and level.

APE1 knockdown abrogates resveratrol-induced neuroprotection against cytotoxicity in OGD/R-treated HT22 cells. Given the obvious increase in DNA repair activity induced by APE1 under OGD/R treatment, we hypothesized that enhancement of DNA repair significantly contributes to RVS-mediated neuroprotection. To confirm this hypothesis directly, we transduced lentiviral vectors containing either shRNA targeted to APE1 (APE1t) or a scrambled control shRNA (APE1s) into HT22 cells. Results from RT-PCR and western blot assasy reveled that the level of APE1 mRNA (Fig. 3A) and protein were (Fig. 3B) decreased in APE1t transfected cells compared to APE1s transfected cells with or without OGD/R treatment, indicating the successful knockdown of APE1 gene in HT22 cells. Next, we found that APE1t transfection remarkably reduced the viability of HT22 cells (Fig. 3C) and increased the LDH leakage (Fig. 3D) compared to RVS pretreatment group, indicating that the down-regulation of APE1 abolished RVS-mediated neuroprotection against OGD/R-induced injuryies in HT22 cells. These dada indicated that APE1 is required for RVS-induced neuroprotection against cerebral ischemia injury.

APE1 knockdown alleviates resveratrol-elicited beneficent effects on oxidative stress under $O G D / R$ condition in $H T 22$ cells. Emerging evidence reveals that APE1 functions in the DNA base excision repair pathway, the redox regulation of several transcription factors, and the control of intracellular redox status through the inhibition of reactive oxygen species (ROS) production. Therefore, we further demonstrated the effect of resveratrol on OGD/R-induced oxidative stress and the role of APE1 in this process. As shown in Fig. 4, resveratrol pretreatment obviously abolished OGD/R-induced the increase in the level of ROS (Fig. 4A). However, this effect was abolished by knockdown of APE1 with APE1t. The antioxidant system has been shown to be crucial for detoxification in many cellular organ systems, so the status of the enzymatic antioxidant superoxide dismutase (SOD) and nonenzymatic antioxidant glutathione (GSH) were evaluated. We found that APE1t transfection remarkably reversed resveratrol-prevented the up-regulation of SOD (Fig. 4B) and GSH activities (Fig. 4C) induced by OGD/R treatment. These results suggested that APE1 contributes to the protective effects of resveratrol against OGD/R-induced oxidative stress, which may be related to attenuation of oxidative stress and enhancement of antioxidant defense.
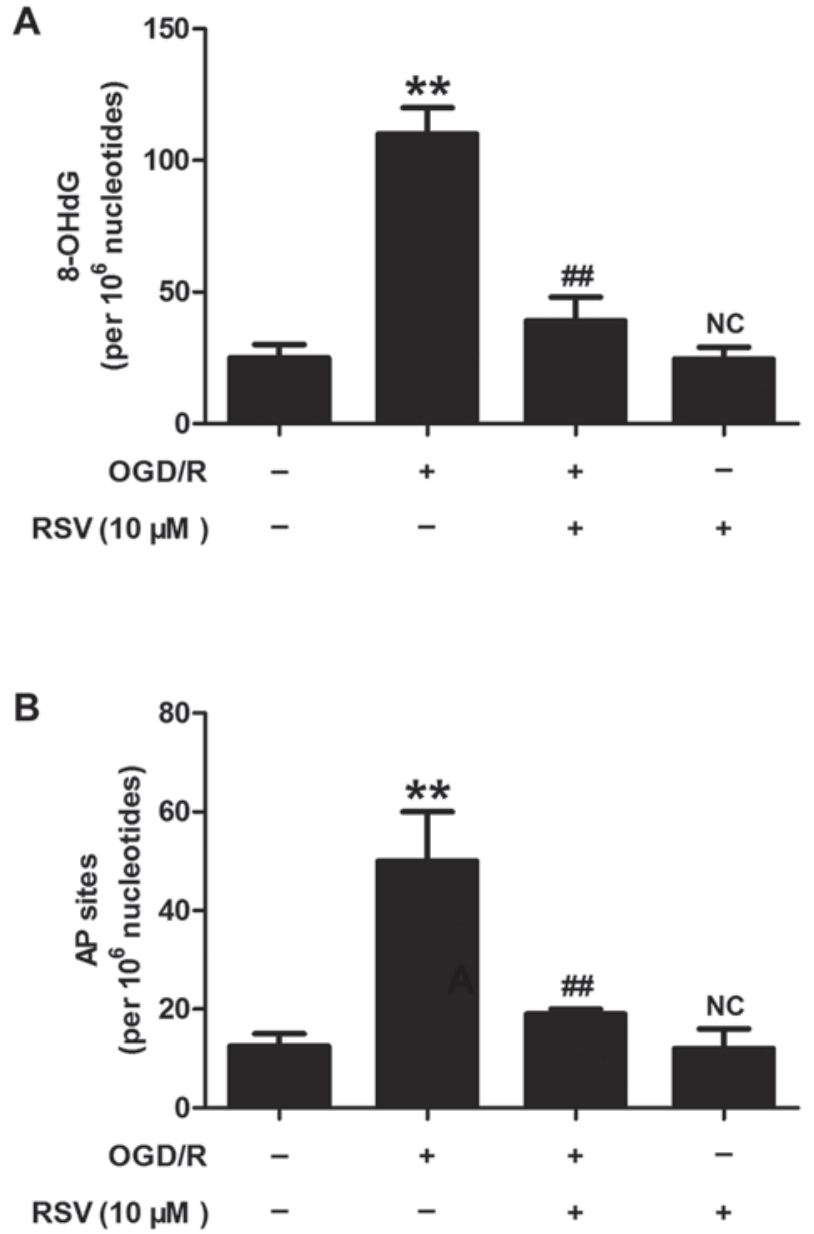

Figure 2. Resveratrol decreases the levels of 8-hydroxy-2ф-deoxyguanosine (8-OHdG) and apurinic/apyrimidinic (AP) sites in OGD/R-treated HT22 cells. HT22 cells were pretreated with resveratrol $(10 \mu \mathrm{M})$ for $30 \mathrm{~min}$ and then subjected to OGD/R treatment. Oxidative DNA damage markers 8-OHdG (A) and AP sites (B) were measured in DNA extracts using colorimetric assay kits, respectively. Bars represent the means \pm SD from three independent experiments. ${ }^{* *} \mathrm{P}<0.01$ vs. control group; ${ }^{\# \#} \mathrm{P}<0.01$ vs. OGD/R treatment alone group. RVS, resveratrol; OGD/R, oxygen-glucose deprivation and re-oxygenation; 8-OHdG, 8-hydroxy-2ф-deoxyguanosine; AP, apurinic/apyrimidinic, NC: No statistical significance.

\section{Discussion}

Hypoxic-ischemic brain injury is an important neurological disorder associated with neonatal death and long-term disability, leading to approximately 6 million deaths every year (23). As the standard of clinical treatment, however, hypothermia has limited utility $(24,25)$. Recent research reveals that APE1-modulated oxidative DNA damage plays an important role in cerebral ischemia injury $(13,26,27)$. Hence, the present study characterizes the beneficent impact of APE1 on resveratrol (a neuroprotective agent)-mediated neuroprotective effect in a cell model of cerebral ischemia injury. Three major findings from this study contribute to our understanding of the role of APE1 in the protective effects of resveratrol against oxygen-glucose deprivation and re-oxygenation (OGD/R)-induced HT22 cells injury. First, resveratrol reversed OGD/R-induced cytotoxicity accompanied by increasing APE1 activity and levels. Second, resveratrol alleviated OGD/R-induced 
A

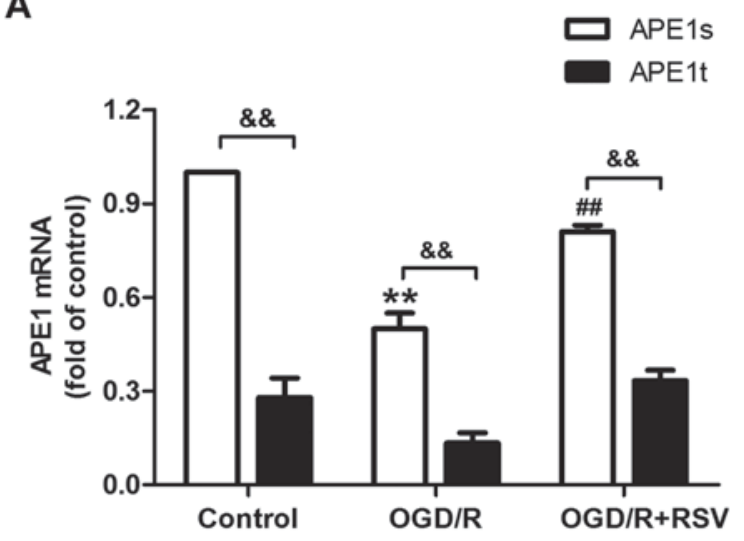

B APE1
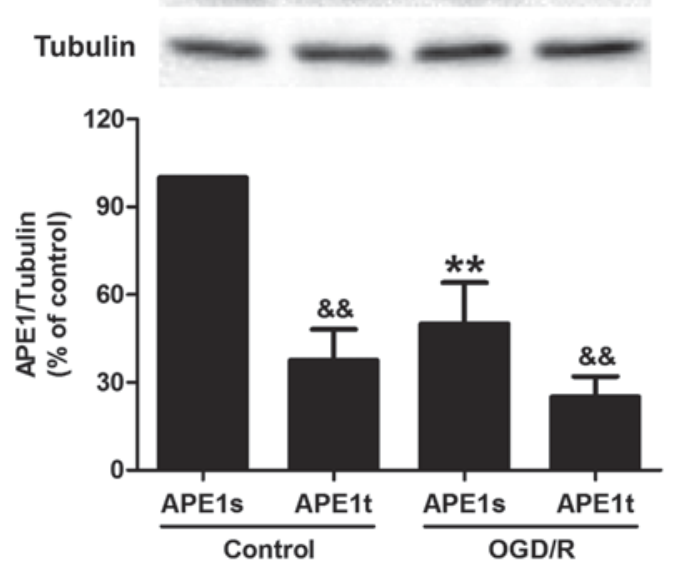

C

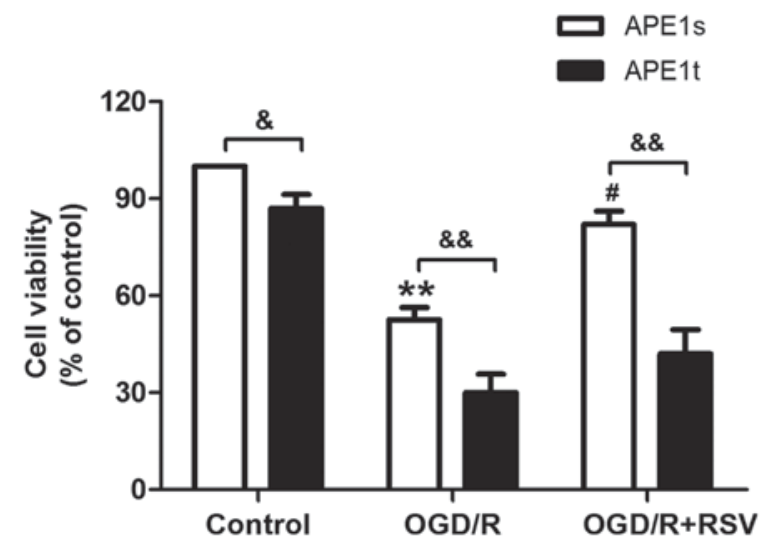

D

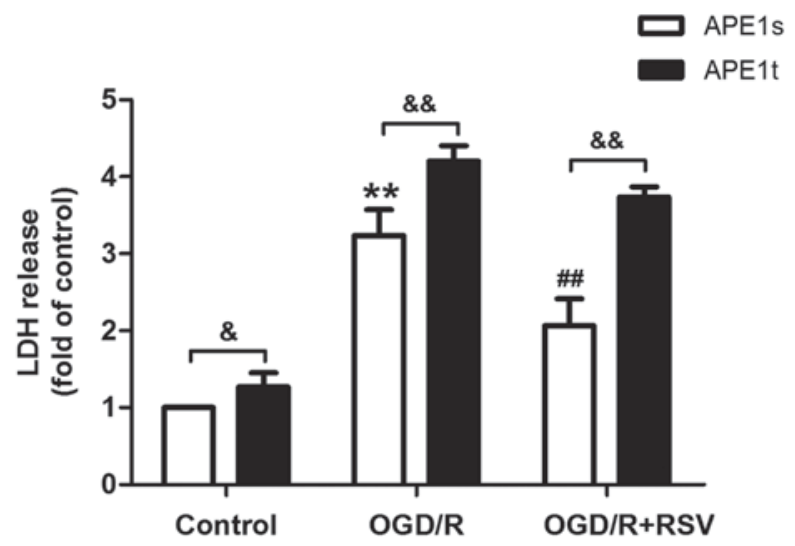

Figure 3. Knockdown of APE1 abrogates resveratrol-induced neuroprotection following OGD/R treatment in HT22 cells. HT22 cells were transfected with lentiviral vectors expressing APE1-targeting sequence (APE1t) or the scrambled sequence (APE1s), and then were treated with resveratrol (10 $\mu \mathrm{M}$ ) for 30 min followed by OGD/R treatment. (A) The level of APE1 mRNA was measured by RT-PCR. Results were normalized to control levels. (B) The expression of APE1 was determined using western blot assay. Densitometry analyses are presented as the relative ratio of protein/tubulin, and data are presented as percentages of control group. (C) The viability of cells were measured by MTT assay. (D) The LDH release was measured by lactate dehydrogenase (LDH) cytotoxicity assay kit. Bars represent the means \pm SD from three independent experiments. ${ }^{* *} \mathrm{P}<0.01$ vs. control group; ${ }^{~} \mathrm{P}<0.05$; ${ }^{\# \#} \mathrm{P}<0.01 \mathrm{vs}$. OGD/R treatment alone group; ${ }^{\&} \mathrm{P}<0.05 ;{ }^{\&} \& \mathrm{P}<0.01 \mathrm{vs}$. RVS and OGD/R co-treatment group. RVS, resveratrol; OGD/R, oxygen-glucose deprivation and re-oxygenation; APE1t, lentiviral vectors containing either shRNA targeted to APE1; APE1s, scrambled control shRNA.

oxidative DNA damage as evidenced by the decreases in the levels of 8 -hydroxy-2 $\varnothing$-deoxyguanosine $(8-\mathrm{OHdG})$ and apurinic/apyrimidinic (AP) sites. Third, APE1 knockdown blocked resveratrol-induced protective effects on cytotoxicity and oxidative stress under OGD/R condition. Our data suggests a promising therapeutic strategy to the cerebral ischemia and reperfusion injury.

Resveratrol, a stilbene formed in many plants in response to various stressors, elicits multiple beneficial effects including anti-oxidative, anti-apoptotic and anti-inflammatory properties in dozens of diseases (28). Particularly, resveratrol was shown to have therapeutic properties in neuronal following ischemia reperfusion injury $(29,30)$. Resveratrol post-treatment protects against neonatal brain injury after hypoxia-ischemia (20). Resveratrol can ameliorate oxidative stress following rat cerebral ischemia-reperfusion injury (31). In present study, we also found that resveratrol also reversed OGD/R-induced neurotoxicity in HT22 cells. Notably, the present show that the concentration of resveratrol $(10 \mu \mathrm{M})$ significantly increased the cell viability compared to resveratrol $(20 \mu \mathrm{M})$, which may be due to that $10 \mu \mathrm{M}$ reaches saturation value of resveratrol and resveratrol may have inhibition on cell viability with the increase of concentration.

Zaky et al confirmed that a reactive oxygen species (ROS)-scavenger, resveratrol, attenuates central inflammation and modulate APE1 expression in aluminum chloride $\left(\mathrm{AlCl}_{3}\right)$-induced neurotoxicity (32). APE1, a multifunctional protein, functions in DNA repair and plays a vital role in cell survival vs. death upon stimulation with cytotoxic agents, making it an attractive emerging therapeutic target (32). Emerging evidence confers the beneficent effects of APE1 on cerebral ischemia. Stetler et al prove that endogenous APE1 protects against ischemic infarction in gray and white matter and facilitates the functional recovery of central nervous system (CNS) after mild stroke injury (26). Leak et al also prove that APE1 up-regulation, either endogenously or through transgene overexpression, reduce oxidative DNA damage and protect hippocampal neurons from ischemic injury (27). In addition, some other studies reveal that APE1 is involved in neuroprotective agents such as $17 \beta$-estradiol (E2) and pituitary 

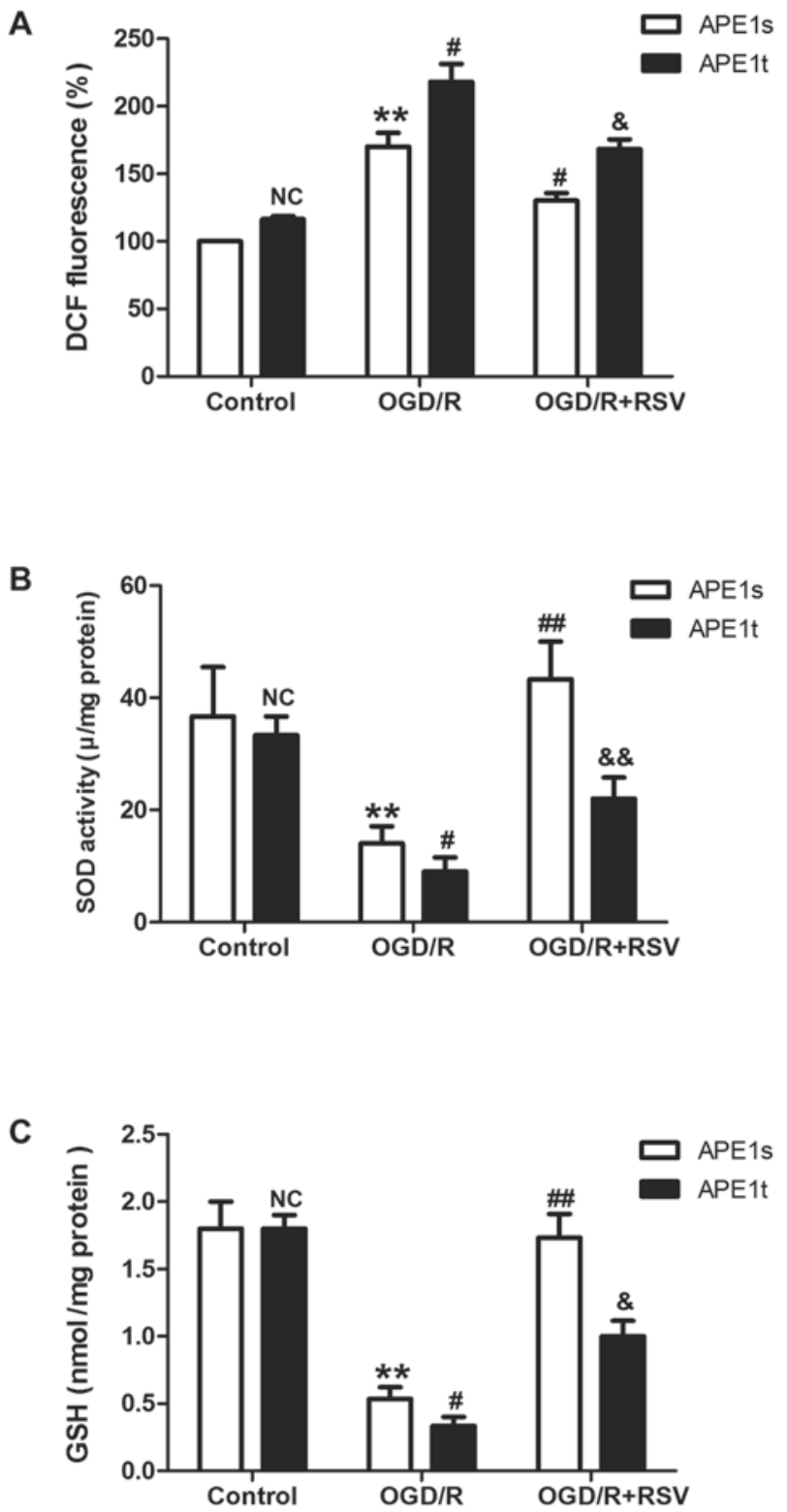

Figure 4. Knockdown of APE1 abolishes resveratrol-induced oxidative stress following OGD/R treatment in HT22 cells. HT22 cells were transfected with APE1t or APE1 s, and then treated with resveratrol $(10 \mu \mathrm{M})$ for $30 \mathrm{~min}$ followed by OGD/R treatment. (A) ROS production was measured by $\mathrm{H}_{2}$ DCF-DA staining. (B) The activity of SOD was measured using a superoxide dismutase (SOD) activity kit. (C) Glutathione quantification was detected using a GSH measurement kit. Bars represent the means \pm SD from three independent experiments. ${ }^{* *} \mathrm{P}<0.01$ vs. control group; ${ }^{\#} \mathrm{P}<0.05 ;{ }^{\# \#} \mathrm{P}<0.01$ vs. OGD/R treatment alone group; ${ }^{\circledR} \mathrm{P}<0.05$; ${ }^{\&} \mathrm{P}<0.01$ vs. RVS and $\mathrm{OGD} / \mathrm{R}$ co-treatment group. RVS, resveratrol; OGD/R, oxygen-glucose deprivation and re-oxygenation; NC: No statistical significance.

adenylate cyclase-activating polypeptide (PACAP) against ischemia-induced damage $(13,33)$. Therefore, we put forward the hypothesis that APE1 may contribute to the neuroprotective effect of resveratrol against cerebral ischemic injury.

Consistent with these studies, in current study, we found that resveratrol pretreatment significantly increased the activity and the level of APE1. A major hallmark of oxidative DNA damage after stroke is the induction of apurinic/apyrimidinic (AP) sites and strand breaks $(4,6)$. APE1 can repair AP sites during base-excision repair (BER) (3). Research has shown that adenovirus-mediated APE1 upregulation reduces 8-OHdG formation, AP sites, DNA fragmentation, and infarct volume after ischemia-reperfusion injury (34). In agreement with these studies, the present study found that resveratrol treatment decreased the level of 8-OHdG and AP sites under ODG/R condition in HT22 cells, further indicating the inconvenient role of APE1 in resveratrol-offered protective effect against oxidative DNA damage in cerebral ischemia reperfusion injury.

Furthermore, we found that APE1 knockdown induced by APE1 shRNA abolished resveratrol-induced the increases in the cell viability and decreases in the LDH release, indicating the contribution of APE1 to the protective effect of resveratrol against OGD/R injury. Increasing evidence confirms that inhibition of oxidative stress of and promotion of antioxidant signaling contributes to neuroprotective agent against ischemia-reperfusion damage to rat brains $(35,36)$. APE1 is a multifunctional protein that plays a vital role in the cellular response to DNA injury and redox regulation against oxidative stress through the inhibition of reactive oxygen species (ROS) production (37). The present study also further showed that APE1 knockdown increased the level of ROS and increased the activity of SOD and GSH compared to OGD/R treatment in HT22 cells. Combined with the previous research related to the relationship between APE1 and oxidative stress, these results suggested that APE1 contributes to the protective effects of resveratrol against OGD/R-induced oxidative stress, which may be related to attenuation of oxidative stress and enhancement of antioxidant defense. However, the protective effect of resveratrol was not completely inhibited by APE1 shRNA. These might be because APE1 shRNA did not completely reduced the APE1 shRNA. In addition, many other studies reveal that resveratrol-activated pathways have been shown to protect against ischemia through modulating SIRT1 activity (38), autophagy (39) and NO signaling (40), further implying that there are further mechanisms involved the protective effects of resveratrol against cerebral ischemia reperfusion injury in independent of APE1 pathway.

Of course, there are many deficiencies in preset article. We discussed the role of APE1 in resveratrol-elicited protective effect in cerebral ischemia injury only in terms of its antioxidant stress activity. Many researches revel that both APE1 and resveratrol have anti-apoptotic and anti-inflammatory activities (41-43), implying that apoptosis may also contribute to the role of APE1 in these. In addition, we only use HT22 cells to test the hypothesis. In the next experiment, we can further investigate the underlying mechanism in in vivo or primary cell models.

In conclusion, in the current study, it was observed that resveratrol significantly decreases OGD/R-induced neurotoxicity through increasing APE1 level and activity. The results further indicate the neuroprotective effects of resveratrol against cerebral ischemia injury are associated with APE1-elicited reduction of oxidative DNA damage involved in attenuation of oxidative stress and enhancement of antioxidant defense. Some other antioxidants such as Picroside II (44), nanomelatonin (45), and A water-soluble polysaccharide (LJPB2) (46) have been proved to protect against cerebral ischemia-reperfusion injury dependent on strong antioxidant capacity. Our study further provide new perspectives that APE1 may also be involved in the neuroprotective effect of these antioxidants. 


\section{References}

1. Roger VL, Go AS, Lloyd-Jones DM, Adams RJ, Berry JD, Brown TM, Carnethon MR, Dai S, de Simone G, Ford ES, et al: Heart disease and stroke statistics-2011 update: A report from the American Heart Association. Circulation 123: e18-e209, 2011.

2. Blakeley JO and Llinas RH: Thrombolytic therapy for acute ischemic stroke. J Neurol Sci 261: 55-62, 2007.

3. Li P, Hu X, Gan Y, Gao Y, Liang W and Chen J: Mechanistic insight into DNA damage and repair in ischemic stroke: Exploiting the base excision repair pathway as a model of neuroprotection. Antioxid Redox Signal 14: 1905-1918, 2011

4. Chen J, Jin K, Chen M, Pei W, Kawaguchi K, Greenberg DA and Simon RP: Early detection of DNA strand breaks in the brain after transient focal ischemia: Implications for the role of DNA damage in apoptosis and neuronal cell death. J Neurochem 69: 232-245, 1997

5. Chen H, Yoshioka H, Kim GS, Jung JE, Okami N, Sakata H, Maier CM, Narasimhan P and Goeders CE,Chan PH: Oxidative stress in ischemic brain damage: Mechanisms of cell death and potential molecular targets for neuroprotection. Antioxid Redox Signal 14: 1505-1517, 2011.

6. Lan J, Li W, Zhang F, Sun FY, Nagayama T, O'Horo C and Chen J: Inducible repair of oxidative DNA lesions in the rat brain after transient focal ischemia and reperfusion. J Cereb Blood Flow Metab 23: 1324-1339, 2003.

7. Dyrkheeva NS, Lebedeva NA and Lavrik OI: AP Endonuclease 1 as a key enzyme in repair of apurinic/apyrimidinic sites. Biochemistry (Mosc) 81: 951-967, 2016.

8. Laev SS, Salakhutdinov NF and Lavrik OI: Inhibitors of nuclease and redox activity of apurinic/apyrimidinic endonuclease 1/redox effector factor 1 (APE1/Ref-1). Bioorg Med Chem 25: 2531-2544, 2017.

9. Liu PK: DNA damage and repair in the brain after cerebral ischemia. Curr Top Med Chem 1: 483-495, 2001.

10. Vasko MR, Guo C and Kelley MR: The multifunctional DNA repair/redox enzyme Ape1/Ref-1 promotes survival of neurons after oxidative stress. DNA Repair (Amst) 4: 367-379, 2005.

11. Ludwig DL, MacInnes MA, Takiguchi Y, Purtymun PE, Henrie M, Flannery M, Meneses J, Pedersen RA and Chen DJ: A murine AP-endonuclease gene-targeted deficiency with post-implantation embryonic progression and ionizing radiation sensitivity. Mutat Res 409: 17-29, 1998.

12. Singh S and Englander EW: Nuclear depletion of apurinic/apyrimidinic endonuclease 1 (Ape1/Ref-1) is an indicator of energy disruption in neurons. Free Radic Biol Med 53: 1782-1790, 2012

13. Stetler RA, Gao Y, Zukin RS, Vosler PS, Zhang L, Zhang F, Cao G, Bennett MV and Chen J: Apurinic/apyrimidinic endonuclease APE1 is required for PACAP-induced neuroprotection against global cerebral ischemia. Proc Natl Acad Sci USA 107: 3204-3209, 2010.

14. Nakata R, Takahashi S and Inoue H: Recent advances in the study on resveratrol. Biol Pharm Bull 35: 273-279, 2012.

15. Yin K, Zhao L, Feng D, Ma W, Liu Y, Wang Y, Liang J, Yang F, Bi C, Chen $\mathrm{H}$, et al: Resveratrol attenuated low ambient temperature-induced myocardial hypertrophy via inhibiting cardiomyocyte apoptosis. Cell Physiol Biochem 35: 2451-2462, 2015.

16. Nie P, Hu W, Zhang T, Yang Y, Hou B and Zou Z: Synergistic induction of erlotinib-mediated apoptosis by resveratrol in human non-small-cell lung cancer cells by down-regulating survivin and up-regulating PUMA. Cell Physiol Biochem 35: 2255-2271, 2015

17. Mokni M, Elkahoui S, Limam F, Amri M and Aouani E: Effect of resveratrol on antioxidant enzyme activities in the brain of healthy rat. Neurochem Res 32: 981-987, 2007.

18. Hung LM, Su MJ and Chen JK: Resveratrol protects myocardial ischemia-reperfusion injury through both No-dependent and NO-independent mechanisms. Free Radic Biol Med 36: 774-781, 2004.

19. Eybl V, Kotyzova D, Cerná P and Koutensky J: Effect of melatonin, curcumin, quercetin, and resveratrol on acute ferric nitrilotriacetate (Fe-NTA)-induced renal oxidative damage in rats. Hum Exp Toxicol 27: 347-353, 2008.

20. Pan S, Li S, Hu Y, Zhang H, Liu Y, Jiang H, Fang M, Li Z, Xu K, Zhang $\mathrm{H}$, et al: Resveratrol post-treatment protects against neonatal brain injury after hypoxia-ischemia. Oncotarget 7: 79247-79261, 2016.
21. Abdel-Aleem GA, Khaleel EF, Mostafa DG and Elberier LK: Neuroprotective effect of resveratrol against brain ischemia reperfusion injury in rats entails reduction of DJ-1 protein expression and activation of PI3K/Akt/GSK3b survival pathway. Arch Physiol Biochem 122: 200-213, 2016.

22. Feng Y, Cui Y, Gao JL, Li MH, Li R, Jiang XH, Tian YX, Wang KJ, Cui CM and Cui JZ: Resveratrol attenuates neuronal autophagy and inflammatory injury by inhibiting the TLR4/NF- $\kappa \mathrm{B}$ signaling pathway in experimental traumatic brain injury. Int J Mol Med 37: 921-930, 2016.

23. Hua C, Ju WN, Jin H, Sun X and Zhao G: Molecular chaperones and hypoxic-ischemic encephalopathy. Neural Regen Res 12: 153-160, 2017.

24. Wu Q, Chen W, Sinha B, Tu Y, Manning S, Thomas N, Zhou S, Jiang $\mathrm{H}$, Ma H, Kroessler DA, et al: Neuroprotective agents for neonatal hypoxic-ischemic brain injury. Drug Discov Today 20: 1372-1381, 2015.

25. Silveira RC and Procianoy RS: Hypothermia therapy for newborns with hypoxic ischemic encephalopathy. J Pediatr (Rio J) 91 ((6 Suppl 1): S78-S83, 2015

26. Stetler RA, Gao Y, Leak RK, Weng Z, Shi Y, Zhang L, $\mathrm{Pu} \mathrm{H}$, Zhang F, Hu X, Hassan S, et al: APE1/Ref-1 facilitates recovery of gray and white matter and neurological function after mild stroke injury. Proc Natl Acad Sci USA 113 E3558-E3567, 2016

27. Leak RK, Li P, Zhang F, Sulaiman HH, Weng Z, Wang G, Stetler RA, Shi Y, Cao G, Gao Y and Chen J: Apurinic/apyrimidinic endonuclease 1 upregulation reduces oxidative DNA damage and protects hippocampal neurons from ischemic injury. Antioxid Redox Signal 22: 135-148, 2015.

28. Lopez MS, Dempsey RJ and Vemuganti R: Resveratrol neuroprotection in stroke and traumatic CNS injury. Neurochem Int 89: 75-82, 2015.

29. Kizmazoglu C, Aydin HE, Sevin IE, Kalemci O, Yüceer N and Atasoy MA: Neuroprotective Effect of Resveratrol on Acute Brain Ischemia Reperfusion Injury by Measuring Annexin V, p53, Bcl-2 Levels in Rats. J Korean Neurosurg Soc 58: 508-512, 2015.

30. Wang R, Liu YY, Liu XY, Jia SW, Zhao J, Cui D and Wang L: Resveratrol protects neurons and the myocardium by reducing oxidative stress and ameliorating mitochondria damage in a cerebral ischemia rat model. Cell Physiol Biochem 34: 854-864, 2014.

31. Li W, Tan C, Liu Y, Liu X, Wang X, Gui Y, Qin L, Deng F, Yu Z, Hu C and Chen L: Resveratrol ameliorates oxidative stress and inhibits aquaporin 4 expression following rat cerebral ischemia-reperfusion injury. Mol Med Rep 12: 7756-7762, 2015.

32. Zaky A, Mohammad B, Moftah M, Kandeel KM and Bassiouny AR: Apurinic/apyrimidinic endonuclease 1 is a key modulator of aluminum-induced neuroinflammation. BMC Neurosci 14: 26, 2013.

33. Dietrich AK, Humphreys GI and Nardulli AM: $17 \beta$-estradiol increases expression of the oxidative stress response and DNA repair protein apurinic endonuclease (Ape1) in the cerebral cortex of female mice following hypoxia. J Steroid Biochem Mol Biol 138: 410-420, 2013

34. Kim HW, Cho KJ, Park SC, Kim HJ and Kim GW: The adenoviral vector-mediated increase in apurinic/apyrimidinic endonuclease inhibits the induction of neuronal cell death after transient ischemic stroke in mice. Brain Res 1274: 1-10, 2009.

35. Li Y and Liu S: The effect of dexmedetomidine on oxidative stress response following cerebral ischemia-reperfusion in rats and the expression of intracellular adhesion molecule-1 (ICAM-1) and S100B. Med Sci Monit 23: 867-873, 2017.

36. Xiao H, Deng M, Yang B, Hu Z and Tang J: Pre-treatment of $17 \beta$-estradiol attenuates cerebral-ischemia-induced blood-brain barrier disruption in aged rats: Involvement of antioxidant signaling. Neuroendocrinology Feb 15, 2017 (Epub ahead of print).

37. Choi S, Joo HK and Jeon BH: Dynamic regulation of APE1/Ref-1 as a therapeutic target protein. Chonnam Med J 52: 75-80, 2016.

38. Howitz KT, Bitterman KJ, Cohen HY, Lamming DW, Lavu S, Wood JG, Zipkin RE, Chung P, Kisielewski A, Zhang LL, et al: Small molecule activators of sirtuins extend Saccharomyces cerevisiae lifespan. Nature 425: 191-196, 2003.

39. Gurusamy N, Lekli I, Mukherjee S, Ray D, Ahsan MK, Gherghiceanu M, Popescu LM and Das DK: Cardioprotection by resveratrol: A novel mechanism via autophagy involving the mTORC2 pathway. Cardiovasc Res 86: 103-112, 2010. 
40. Malhotra A, Bath S and Elbarbry F: An organ system approach to explore the antioxidative, anti-inflammatory and cytoprotective actions of resveratrol. Oxid Med Cell Longev 2015: 803971, 2015.

41. Thakur S, Sarkar B, Cholia RP, Gautam N, Dhiman M and Mantha AK: APE1/Ref-1 as an emerging therapeutic target for various human diseases: Phytochemical modulation of its functions. Exp Mol Med 46: e106, 2014.

42. Xu Y, Zhang B, Xie D, Hu Y, Li HL, Zhong LL, Wang HW, Jiang W, Ke ZP and Zheng DH: Nanoparticle-mediated dual delivery of resveratrol and DAP5 ameliorates kidney ischemia/reperfusion injury by inhibiting cell apoptosis and inflammation. Oncotarget 8: 39547-39558, 2017.

43. Kolahdouz Mohammadi R and Arablou T: Resveratrol and endometriosis: In vitro and animal studies and underlying mechanisms (Review). Biomed Pharmacother 91: 220-228, 2017.
44. Zhai L, Liu M, Wang T, Zhang H, Li S and Guo Y: Picroside II protects the blood-brain barrier by inhibiting the oxidative signaling pathway in cerebral ischemia-reperfusion injury. PLoS One 12: e0174414, 2017.

45. Sarkar S, Mukherjee A, Das N and Swarnakar S: Protective roles of nanomelatonin in cerebral ischemia-reperfusion of aged brain: Matrix metalloproteinases as regulators. Exp Gerontol 92: 13-22, 2017.

46. Su D, Li S, Zhang W, Wang J, Wang J and Lv M: Structural elucidation of a polysaccharide from Lonicera japonica flowers and its neuroprotective effect on cerebral ischemia-reperfusion injury in rat. Int J Biol Macromol 99: 350-357, 2017. 